\title{
External Ion Injection into an EBIS source: Efficiency Measurements
}

\author{
E. Beebe \#, L. Liljeby and A. Pikin \\ Manne Siegbahn Laboratory, S-10405 Stockholm, Sweden \\ E.D. Donets \\ Joint Institute for Nuclear Research, 141980 Dubna, Russia \\ D. Habs \\ Max Planck Institut, D-69029 Heidelberg, Germany \\ K. Janko $\infty$, O. Tengblad, P. Van Duppen and the ISOLDE \\ collaboration \\ CERN-ISOLDE, CH-1211 Geneva 23, Switzerland
}

Abstract

A concept for a charge state multiplier, to be used for the production of energetic radioactive ion beams based on the ISOL+post-accelerator system, is discussed. The charge state breeder implies external ion injection into an Electron Beam Ion Source (EBIS). The total efficiency, measured by injecting a low-energy nitrogen $\left(\mathrm{Q}=1^{+}\right)$beam, reached $14.5 \%$ and was essentially constant for confinement times up to $200 \mathrm{~ms}$. 
$\infty)$ Permanent address: Faculty of Mathematics and Physics, Comenius University, Bratislava, Slovakia

\#) Present address: Brookhaven National Laboratory (AGS dept.) Upton, N.Y. 11973, U.S.A. 
During the last decade a vast interest has developed for the use of energetic radioactive beams for experiments in widely different fields of physics [1]. These beams can be produced in two ways: 1. fragmentation of high-energy stable beams whereby the kinematics of the reaction products is used to obtain a pure secondary beam, and 2. post-acceleration of low energy radioactive ion beams produced at an on-line mass separator. In the latter scenario the production of a low-energy beam of short lived radioactive nuclei is one of the main issues. This production process has to be fast, efficient, and preferentially element selective. Present day development programs at on-line mass separators are dealing with these problems [2,3] and a comparison between the different production and ionization methods can be found in ref [4]. But as the simplicity, efficiency and cost of the acceleration stage is directly related to the charge state of the low energy ions, it is of extreme importance to produce multiply charged ions before injection into the post-accelerator. Typical charge to mass ratios (Q/A) that are needed vary between $1 / 9$ and $1 / 4$. There are basically three types of ion sources which can readily produce highly charged ions: Electron Cyclotron Resonance (ECR) ion sources [5], Electron Beam Ion Sources (EBIS) [6] and laser ablation sources [7]. While the latter type is still in a stage of development, the ECR and EBIS systems are both used for injecting ion beams of stable isotopes into accelerators or storage rings. Recently, ECR sources were installed at on-line mass separators for the efficient production of gaseous elements (like nitrogen, carbon, oxygen and neon) also in higher charge states [8]. Radioactive Ion Beam projects 
(RIB), where an ECR source is coupled with a production target in the high radiation environment of the separator front-end, are proposed at some facilities. A variation of this scheme, in which the target is coupled by a long transfer line to the ECR source, is operational at Louvain-la-Neuve and produces radioactive beams of various gaseous elements (energy between 0.65 and $5.0 \mathrm{MeV} / \mathrm{u}$ ) [9]. Proposals for coupling an EBIS source to a production target have not yet been presented. A few years ago, a radically different idea evolved whereby an EBIS could be used as an ion charge multiplier $[10,11]$. A low-energy $1^{+}$ion beam from an on-line mass separator is injected and trapped in the EBIS source, and then extracted as a pulse of multiply charged ions. The advantage is that, for the production of the low-energy $1^{+}$ion beam, one can rely on the vast experience that is present at on-line mass separators, such as ISOLDE at CERN [12]. Furthermore one avoids the installation of sophisticated equipment - such as high charge state ion sources - in the rather hostile high-radiation environment present at the frontend of an on-line separator.

External ion injection, sometimes including isotope separation, has been developed as an alternative to gas injection into EBIS [13, 14]. The benefits include: decreased gas load in the ionization region, an increased range of injectable elements, and isotopically pure injection which leads to increased intensities of certain elements. For EBIS and other ion sources used as injectors for accelerators the peak current or the total number of ions in the pulse are optimized. This is in contrast to sources used at on-line mass separators where the ionization efficiency is optimized [3]. 
Consequently, there has been a paucity of data about the efficiency of an EBIS system using external ion injection. In this letter we report on efficiency measurements using the EBIS system (CRYSIS) and the isotope separator (INIS) of the Manne Siegbahn Laboratory (MSL) in Stockholm [15].

A nitrogen ion beam was extracted from a Unoplasma ion source and momentum analyzed at $25 \mathrm{QxkV}$ (Q is the charge state of the ions). The selected $\mathrm{N}^{+1}$ ions were decelerated to $3.5 \mathrm{keV}$ before entering the EBIS beam line. Measurements were made in the "fast" or "pulsed" injection mode, in which the primary ions are accepted by the EBIS during a relatively brief injection time, $t_{i n j}$. This is accomplished by suitably lowering the potential applied to one of the axial electrostatic trap barriers. In CRYSIS, ion injection and subsequent ion extraction are made across the same barrier, called the extraction barrier. A more positive potential is maintained on the gun-end barrier to prevent ions from draining towards the electron gun. Ions are confined within the EBIS for further ionization by the electron beam during a period $\mathrm{t}_{\mathrm{conf}}$, typically 1-1000 ms. Ions are expelled over the extraction barrier by rapidly increasing the applied trap region potential, $\mathrm{V}_{\mathrm{trap}}$, to a value greater than that of the extraction barrier potential, $\mathrm{V}_{\text {ext }}$.

Efficiency values for the process are deduced from current measurements of the injected and extracted beams, in conjunction with the time of flight observations to determine the ratio of the extracted charge states. The injected beam of $\mathrm{N}^{+1}$ is monitored on a plate inserted intermittently into the beam path close to the exit of the EBIS. The extracted EBIS beam is monitored on an off-axis 
faraday cup to avoid blocking the primary (injected) beam. During the measurements reported, a $1.2 \mathrm{~mA}$ beam of $\mathrm{N}^{+1}$ ions was injected into the one meter long ion confinement region of the EBIS. Trapping and further ionization was made within the EBIS by a 250 $\mathrm{mA}, 16 \mathrm{keV}$ electron beam. Table 1 gives the values of the source parameters in the different phases of the process. The optimum value for $t_{i n j}$ was deduced to be $100 \mathrm{~ms}$ i.e. 120 particle pico Coulomb (ppC) per pulse were injected into the source: $100 \mathrm{~ms}$ corresponds to a traveling distance of about $2.5 \mathrm{~m}$ for a $14 \mathrm{~N}+1$ ion having an energy of $40 \mathrm{eV}$. The charge state distribution was determined by sampling part of the output pulse for $200 \mathrm{~ns}$ with a TOF spectrometer. Examples of some TOF spectra are shown in figure 1 with $\mathrm{t}_{\mathrm{conf}}$ equal $1.5 \mathrm{~ms}, 2.5 \mathrm{~ms}$ and $200 \mathrm{~ms}$. Note that after $200 \mathrm{~ms}$ about $60 \%$ of all the trapped nitrogen ions are fully stripped. For short confinement times, thus low-charge states, slight adjustments of the TOF spectrometer changed the apparent charge state distribution. For the determination of the absolute distribution the TOF spectrometer was optimized for every charge state separately. Figure 2.a shows the total extracted charge in the pulse as a function of confinement time. After correction for the charge state distribution, the extracted number of ions per pulse versus the confinement time is obtained (Fig. 2.b). The total efficiency defined as the number of ions extracted from the EBIS per pulse divided by the number of ions injected into the EBIS from the external source per pulse- is derived from current measurements near the EBIS exit and the injection time. One notices that the extracted number of particles per pulse is essentially constant, i.e. 
independent of the confinement time at least up to $\mathrm{t}_{\mathrm{conf}}=200 \mathrm{~ms}$. We obtain a total efficiency of $14.5 \%$ and as an example one can quote a $7 \%$ efficiency for the production of a $3^{+}$charge state after $1.5 \mathrm{~ms}$ confinement time and a $9 \%$ efficiency for the production of fully stripped nitrogen ions after $\mathrm{t}_{\mathrm{conf}}=200 \mathrm{~ms}$.

Observations made during several trails show that achieving sufficient overlap between the injected ion beam and the electron beam is crucial for obtaining reasonable efficiencies and narrow extracted charge state distributions. The ion losses of the charge breeding procedure are entirely due to the injection process as the total efficiency does not depend on the confinement time (at least up to $200 \mathrm{~ms}$ ). An estimate of the total efficiency can be obtained by comparing the phase space acceptance of the electron beam with the phase space emittance of the INIS ion beam (normalized emittance between 1.2 and $3.410^{-8} \mathrm{p} \mathrm{m} \mathrm{rad}$ ). In this way we obtain a value, between 8 and $71 \%$, that covers our experimental efficiency $(14.5 \%)$. From the charge state distribution we can calculate the effective electron beam current density using a batch model type of calculation with ionization cross sections for nitrogen obtained from reference [16]. The model assumes that the ions are confined $100 \%$ of the time within the electron beam, a condition which is probably not met for externally injected ions even shortly after injection [17]. For short confinement times $\left(\mathrm{t}_{\mathrm{conf}}=1.5\right.$ and $2.5 \mathrm{~ms}$ ) we obtain about $140 \mathrm{~A} / \mathrm{cm}^{2}$ but for $\mathrm{t}_{\mathrm{conf}}=200 \mathrm{~ms}$ the effective electron current density dropped to $30 \mathrm{~A} / \mathrm{cm}^{2}$. This effect could be due to a further reduction in overlap of the ion beam with the electron beam induced by heating or by space charge effects. 
Interesting to note is that problems related to the physicochemical properties of specific elements are of no concern to the system proposed as the particles are treated as free ions during the whole breeding process. This is in contrast to other ion sources in which sticking to the walls of the source causes losses in total efficiency due to the decay of the wanted short lived radioactive nuclei [9]. Apart from the efficiency we should also consider the duty cycle overlap between the primary $1^{+}$beam and the injection timing of the EBIS system. The repetition rate $\left(n_{r e p}\right)$ is defined as the inverse of the sum of the confinement, injection, extraction and cleaning periods of the source. Taking for the latter a realistic number of $0.5 \mathrm{~ms}$ one obtains the following relation: $\mathrm{n}_{\mathrm{rep}}=$ $1 /\left(0.0005+t_{c o n f}\right)$. Thus the primary beam of radioactive ions has to be delivered in bunches (i.e. all the radioactive ions are squeezed into pulses without losses) with frequency equal to $n_{r e p}$ and pulse duration less than $t_{i n j}$ (e.g. $100 \mathrm{~ms}$ in the case given). These conditions can, for a limited number of elements, be fulfilled with a surface ion source or a laser ion source using a positively biased electrode just in front of the exit hole $[18,19]$. Bunched beams can in principle also be created by laser induced ablation of the radioactive atoms that are condensed on a cooled spot in the ion source [20] or by capturing the $1^{+}$ions in a Paul Trap Ion Buncher [21]. Alternatively, an EBIS can also be used to accumulate a continuous beam of radioactive ions [11]. In this "slow" or continuous mode, ions are injected over an extraction barrier which is held at a more positive voltage than the trap region, but typically more negative than the gun-barrier potential. Injected ions which 
reach the trap region and are at least partially confined within the high density electron beam, have a high probability of being ionized to higher charge states, and thereby becoming trapped even by a fixed extraction barrier. The primary $1^{+}$ions are not accepted during the extraction and cleaning periods and continuous injection results in a broader charge state distribution than pulsed injection.

In conclusion, we have presented results from efficiency measurements with an EBIS system using external ion injection. These measurements have shown that an EBIS system can be used as an efficient charge state breeder for the $1^{+}$radioactive ion beams produced by an on-line mass separator. Since the demanded Q/A ratio for a "standard" post-accelerator is modest (no fully stripped ions are requested) and since the primary radioactive ion beam intensity is limited - especially for nuclei far from the line of stability - the EBIS system is used well below its performance limits. The modest Q/A ratio requires relatively brief confinement times hence losses due to heating of the ions by inelastic electron scattering are small in our proposed system. We are often dealing with a rather small number of injected ions so that the ion trap will be filled to a small fraction of its maximum capability. The Stockholm system was developed to maximize the output of highly charged ions rather than efficiency as described in this context. Thus the efficiency values obtained can be regarded as conservative estimates. It is expected that the efficiency can be substantially improved in an EBIS-based system designed and dedicated to such a task. The scheme presented here for coupling a post-accelerator to an on-line isotope separator through an EBIS-system placed in the 
experimental area, forms an interesting alternative to other schemes for the production of energetic radioactive ion beams. The reported high efficiencies for the production of multiply charged ions make it worthwhile to pursue this development as the question about the efficiency has so far always been a major obstacle. 


\section{$\underline{\text { References: }}$}

1. Th. Delbar (editor), Proc. of the Second Int. Conf. on Radioactive Nuclear Beams, Louvain-la-Neuve (Belgium) (1992) Adam Hilger, New York

2. H.L. Ravn and B.W. Allardyce, in "Treatise of Heavy Ion Science", Ed. D.A. Bromley, (Plenum Press New York) 8 (1989) 363

3. P. Van Duppen, P. Decrock, M. Huyse and R. Kirchner, Rev. Sci. Instrum. $63 \quad(1992) 2381$

4. H.L. Ravn et al., Nucl. Instr. and Meth. accepted for publication 5. R. Geller, VI Conference on the Physics with Highly Charged Ions, AIP conf. 274, Manhatten Kanses, U.S.A., 1992

6. E.D. Donets, in "The Physics and Technology of Ion Sources", Ed. I.G. Brown, 12 (1989) 245 (Wiley, New York)

7. T.R. Sherwood, Rev. Sci. Instrum. 63 (1992) 2789

8. P. Decrock, M. Huyse, P. Van Duppen, F. Baeten, C. Dom and Y. Jongen, Nucl. Instr. and Meth. B58 (1991) 252

9. P. Decrock et al., Phys. Rev. Lett 67 (1991) 808

10. H. Haas, H. Ravn, A. Schempp, B.W. Allardyce, B. Jonson, C. Rolfs and the ISOLDE Collaboration, , Proc. of the First Int. Conf. on Radioactive Nuclear Beams, Berkeley (U.S.A.) Ed. W.D. Myers, M. Nitschke, E.B. Norman (1990) 59 (World Scientific)

11. R. Becker, M. Kleinod, J.W. Goethe, E.D. Donets and A.I. Pikin, Third European Particle Accelerator Conference (EPAC-92), Editions Frontières, Ed.: H. Heinke, H. Homeyer and Ch. Petit-Jean-Genaz (1992) 981 
12. E. Kugler, D. Fiander, B. Jonson, H. Haas, A. Przewloka, H.L. Ravn, D.J. Simon and K. Zimmer, Nucl. Instr. and Meth. B70 (1992) 41

13. J. Faure, B. Feinberg, A. Courtois and R. Gobin, Nucl. Instr. and Meth. 219 (1984) 449

14. L. Liljeby and A. Engström, Int. Symposium on Electron Beam Ion Sources and their Applications, AIP nr. 188, N.Y. (1988)

15. E. Beebe. L. Liljeby, A. Engström and M. Björkhage, Physica Scripta 47 (1993) 470

16. A. Müller, E. Salzborn, R. Frodl, R. Becker, H. Klein and H. Winter, Journal of Physics B: Atomic and Molecular Physics 13 (1980) 1877

17. J. Axelsson, MSI Report ISSN-1100-214X (1990)

18. Y. Shirakabe, N. Ikeda, S. Ohkawa, T. Nomura and T. Shinozuka, Nucl. Instr. and Meth. A337 (1993) 11

19. V.I. Mishin, V.N. Fedoseyev, H.J. Kluge, V.S. Letokhov, H.L. Ravn, F. Scheerer, Y. Shirakabe, S. Sundell, O. Tengblad and the ISOLDE Collaboration, Nucl. Instr. and Meth. B73 (1993) 550

20. H.L. Ravn, Nucl. Instrum. and Meth. B70 (1992) 107

21. R.B. Moore and G. Rouleau, Journal Mod. Opt. 39 (1992) 361 
Figure Captions:

Figure 1: Time of Flight (TOF) spectra with a $\mathrm{t}_{\mathrm{conf}}$ equal $1.5 \mathrm{~ms}, 2.5$ $\mathrm{ms}$ and $200 \mathrm{~ms}$. For short confinement times the TOF parameters were adjusted according to the charge state of interest. The TOF spectrum with $\mathrm{t}_{\mathrm{conf}}$ equal $1.5 \mathrm{~ms}$ and $2.5 \mathrm{~ms}$ were optimized for charge state $2^{+}$and $4^{+}$respectively.

Figure 2: The total extracted charge per pulse (a) and the total extracted number of ions per pulse (b) as a function of the confinement time. 
Table captions:

Table 1: The main source parameters used during the different phases of the experiment. The confinement time $\left(t_{c o n f}\right)$ was varied between $1.5 \mathrm{~ms}$ and $200 \mathrm{~ms}$. The ions $\left(\mathrm{Q}=1^{+}\right)$were injected with an energy of $3500 \mathrm{eV}$.

\begin{tabular}{|c|c|c|c|}
\hline & $\mathrm{V}_{\text {trap }}(\mathrm{V})$ & $\mathrm{V}_{\text {ext }}(\mathrm{V})$ & $\mathrm{t}(\mathrm{ms})$ \\
\hline & & & \\
\hline Injection & 3460 & 3390 & 0.100 \\
\hline Confinement & 3460 & 3530 & $\mathrm{t}_{\mathrm{conf}}$ \\
\hline Extraction & 3680 & 3530 & 0.5 \\
\hline Cleaning & 3540 & 3530 & 11.0 \\
\hline
\end{tabular}

